\title{
US to "rethink" Ebola infection control after nurse falls ill
}

\author{
Michael McCarthy
}

Seattle

The director of the US Centers for Disease Control and Prevention, Thomas Frieden, said on 13 October that the CDC was conducting an "in-depth review and investigation" to determine how a Texas nurse who took care of a Liberian man with Ebola became infected with the virus.

"We have to rethink the way we address Ebola infection control, because even a single infection is unacceptable," Frieden said at a press briefing.

The nurse, 26 year old Nina Pham, of Fort Worth, Texas, had extensive contact with the patient, Thomas Eric Duncan, a 42 year old Liberian man who contracted the disease while in west Africa. ${ }^{1}$ Duncan did not fall ill until after he had arrived in Texas. He was admitted to Texas Health Presbyterian Hospital in Dallas on 25 September. Despite receiving ventilator support, renal dialysis, and treatment with an experimental antiviral, brincidofovir, an oral nucleotide analog that has been shown to be active against the virus in vitro, he died 8 October. ${ }^{2}$

Pham, who had been monitoring herself for symptoms, reported that she had developed a low grade fever and on 10 October was admitted to Texas Health Presbyterian Hospital, where she was put into isolation. Laboratory tests conducted over the weekend confirmed that she had contracted the virus. Pham is reported to be in a clinically stable condition.

Investigators have not determined how Pham, who wore personal protective gear while caring for Duncan, became infected, Frieden said. However, her infection raised concern that others who cared for Duncan while he was in hospital may have been exposed as well, he said. "We're concerned and would unfortunately not be surprised if we did see additional cases in the healthcare workers who also provided care to the index patient." All hospital personnel who were involved in Duncan's care are being monitored for signs of infection.

CDC investigators will be looking at the infection control protocols that the hospital workers followed before they entered the isolation unit, while they were with the patient in the unit, and after they left the unit, Frieden said. Investigators are particularly concerned about protocols the staff followed while removing their personal protective gear. "If it is contaminated, there's a possibility that a worker will contaminate themselves and become infected in that process," Frieden said. The CDC is considering recommending that healthcare workers spray their protective gear with a decontaminating product before disrobing.

CDC investigators are also concerned that healthcare workers may be unnecessarily adding extra layers of gloves and protective gear in the belief that the additional layers will provide more protection. "Healthcare workers may think that more is better, so may put on additional sets of gloves or additional coverings, and that may actually end up paradoxically making things less rather than more safe because it may be so difficult to remove those levels or layers that it inadvertently increases risk," Frieden said.

To improve the US health system's response to the Ebola epidemic, US health officials "will double down on training, outreach, education, and assistance throughout the healthcare system, through professional associations, through hospitals, through group organizations and individuals," Frieden said.

"The single most important thing for every other hospital in the country to know is the importance of taking a history of travel-that if someone has fever or other symptoms that could be Ebola, ask where they have been in the previous 21 days. And if it's to Liberia, Sierra Leone, or Guinea, then immediately place them in isolation, consult with us, the state and local health department, and we'll go from there," Frieden said.

Frieden said he remained confident that the US health system could stop the spread of the virus, noting that doctors in the US and abroad, including the charity Doctors Without Borders (Médecins Sans Frontières), had taken care of patients with Ebola safely. "We know how to do it," he said.

McCarthy M. Texas healthcare worker is diagnosed with Ebola. BMJ 2014;349:g6200 2 McCarthy M. Liberian man being treated for Ebola in Texas dies. BMJ 2014;349:g6145.

Cite this as: BMJ 2014;349:g6240

๑๑ BMJ Publishing Group Ltd 2014 